\title{
Broadband Internet Access Services through Power Line Communication to the Rural Dwellers in Nigeria
}

\author{
Femi-Jemilohun Oladunni Juliet \\ Department of Electrical and Electronic Engineering \\ Ekiti State University, Ado Ekiti \\ Ekiti State, Nigeria
}

\begin{abstract}
The Nigerians in the rural areas are the most hit by the shortage of internet facility even though there is a proliferation of same in the recent time. This work proposes the power line communication as a means to bridge this gap by bringing internet connectivity to homes, offices and factories to a large percentage of the population through the already established power line networks. At the moment about $50 \%$ of the population are duly connected to the power grid (while conscious efforts is on the way by Government to increase electricity accessibility of its citizenry to $80 \%$ in the near future). This suggests that if PLC is implemented, half of the nation population will have access to internet service at their door steps. Various challenges in the different and previous technologies of internet connections have opened up PLC as a super high way for wide bandwidth potential for multimedia application for the end users.
\end{abstract}

Keywords - Internet, connectivity, power grid, Power line communication, rural areas, mulitmedia.)

\section{INTRODUCTION}

The advent and proliferation of internet in the recent times has increased the quest for digital telecommunications services in every dwelling places of human beings. One would have thought that the spread of wireless telecommunication in Nigeria would make internet access much easier and simpler to every Nigerian who desires it; however, almost two decades after the launch of mobile telephones, quality internet access still eludes most Nigerians.

Out of the huge population of about 180 million people in Nigeria, only $27 \%$ of this has accessibility to internet service, though record has it that Nigeria has the highest internet subscribers in the Africa and comes 20 in ranks in the globe. A large percentage of Nigeria citizen dwells in the remote areas where the presence and availability of basic amenities such as telephony, internet provision is still a mirage. Broadband access is likely going to be the only way out of connectivity shortage and quick connection to internet services which will integrate data, voice and video service applications for the benefits of rural and remote dwellers of our great nation [1].

The importance of communication between the rural dwellers with themselves as well as with urban dwellers to satisfy the vital part of social, economic and political daily living, at very high and effective internet access will be required to achieve this. The major challenge has been the absence of adequate infrastructure for the different digital telecommunication systems that have emerged over the years to provide the internet services at various degree of data rates to populous country like ours. Due to some technical challenges of these various technologies at different levels, internet access has not been able to go round for every citizenry especially people in the remote part of the country. The possibility of digital telecommunication services being super impose on the already existing electricity distribution networks will provide a superhighway for broadband internet connectivity to every home, office, factory and a large percentage of the nation if not all will the connected regardless of their location in the country [2].

Broadband internet technology has the potential of higher capacity delivery of internet access with wider coverage. The existing wired network has the challenge of high cost for the rural dwellers to afford. Wireless network that would have been the solution is also faced with penetration hitches when line of sight is not sustained. The way out will be the broadband access using the solid infrastructure of the power sector to access potential and competence with low cost , making the delivery of ubiquitous, high data rate connectivity a reality for all and sundry in the nation. The power line communication will without doubt offer this desire of the citizen for ease access of internet network services.

Power Line Communication (PLC) is a communication technology that enables sending data over existing power cables. This means that, with just power cables running to an electronic device, one can simultaneously power it up and retrieve data from it in a half-duplex manner [3]. The PLC networks has been found to compete favorably with WiFi and domestic Ethernet in terms of high data rates delivery capability. This qualifies this technology for multimedia applications that take place in homes and offices. The research trend lately is focusing the engaging power line network operation at $1 \mathrm{MHz}$ for telecommunication with assurance of data rates delivery to the several megabits in a second [3].

\section{Classes of PLC}

The two major classes according to $[4,5]$ are

(i) Narrowband PLC: Narrowband PLC works at lower frequencies $(3-500 \mathrm{kHz}$ ), lower data rates (up to $100 \mathrm{~s}$ of kbps), and has longer range (up to several kilometers), which can be extended using repeaters. Recently, narrowband Power Line Communication has been receiving widespread attention due to its applications in the Smart Grid. Another application that narrowband PLC has been used in is smart energy generation, particularly in micro-inverters for solar panels.

(ii) Broadband PLC: Broadband PLC, in contrast, has mainly found acceptance as a last-mile solution for Internet 
distribution and home networking. Broadband PLC works at higher frequencies (1.8-250 MHz), high data rates (up to 100s of Mbps) and is used in shorter-range applications. With its high data rates and no additional wiring, broadband PLC is seen as an exciting and effective technology for multimedia distribution within homes.

\section{Electricity Distribution in Nigeria}

Table 1 shows the electricity distribution in the six Geopolitical zones of Nigeria. Electricity being a major factor to the growth and development of social and economic life of any nation both at micro and macro levels, the statistics as revealed by the table shows that there is still much work to be done to get electricity to the far and remote places of the country. Based on this, Nigeria Government mandated himself to acquire $80 \%$ electricity coverage by 2015 as part of agenda of the power sector. Though three years after, this has not been achieved but there is a considerable improvement in power distribution across the country. It is also certain that the government is concerned in the provision of electricity to numerous number of citizen in the rural communities as well as those that are distant from the existing national grid [6].

One of the ways to achieve this is to explore the off-grid electrification scheme. Consideration is on the by the World Bank to engage off-grid electrification techniques to provide electricity to the small, low-income earners in the rural communities [6]. If this is achieve, then the idea of PLC will become a reality to reach the rural dwellers with broadband access

\section{LITERATURE REVIEW}

The Emergence of PLC has given birth to home network technology where there is room for the broadband network system to piggy back on the already existing electricity distribution networks for the purpose of data transmission and access connectivity of home appliances to one another and to the internet. The installation of this is highly cost effective and its ubiquity as long as electric power outlet is available makes it more attractive more than other communication systems. Though the data transmission over energy network has been around for quite a while, the explosive proliferation of internet as well as unprecedented rise in the Very Large SemiConductor Integration (VLSI) and Digital Signal Processing (DSP) technology heralded by the deregulation of the telecommunication sector in US, Europe and Asia in the 1990s. This has aroused the interest of both researchers and end users in the merger of broadband access and power line grids for delivery of wide bandwidth for real time application of multimedia services even to the rural dwellers of the Nation [7].

TABLE 1: ELECTRICITY DISTRIBUTION IN THE SIX GEOPOLITICAL ZONES OF [6]

\begin{tabular}{|l|l|}
\hline \multicolumn{1}{|c|}{ Zones } & \multicolumn{1}{c|}{ Electricity Distribution (\%) } \\
\hline North -West & 40.6 \\
\hline North-Central & 40.5 \\
\hline North-East & 28.4 \\
\hline South-West & 74.9 \\
\hline South-East & 59.8 \\
\hline South-South & 65.9 \\
\hline
\end{tabular}

Power Line Communication (PLC) technologies maximize the use of existing power grid as a communication for pointpoint network line for the efficient transmission of data and control of signal. This will enhance smartness across power grid and home automation for the system efficacy. PLC is like any other communication technology whereby a sender modulates the data to be sent, injects it onto medium, and the receiver de-modulates the data to read it. The major difference is that PLC does not need extra cabling, it re-uses existing wiring [8].

\section{Power Line Communication in Nigeria}

Although the first carrier frequency systems began to operate over high-tension lines with frequencies of 15 to 500 $\mathrm{kHz}$ for telemetry purposes in 1922, and this continues and consumer products such as baby alarms have been available at least since 1940, the implementation of PLC in Nigeria is still absent or at least not public. However, there are areas where PLC could be beneficial to the nation's drive towards internet access for all who desire it. Broadband communication over power line could be initiated to provide point-to-point connections. This allows dual data transmission over existing medium voltage (MV) and low voltage (LV) wiring between transformer and customer outlets typically $180 \mathrm{~V}-240 \mathrm{~V}$. The ability to integrate "intelligent" power line networks will support use of real-time supervisory control and data acquisition (SCADA) devices and knowledge acquisition. This will provide market perspectives and promising applications to assess the viability of communications environment [4, 9, and 10].

\section{OPERATIONAL METHODOLOGY}

In achieving the goal of power line communication, the PLC modems serve as interface between the power network and the electronic appliances. Data signal from conventional communication devices, (computer, and telephone) is converted by PLC modem to a form that is suitable for transmission over power lines. It was discovered that the newly wave propagation mechanism in the microwave spectrum called E-line has a great capacity for high speed data rate, though it was only deployed on a single phase of the power line. This establishes the fact of the great potential and excess of gigabit/s data rate delivery when it is deployed at full duplex communication [7].

The deployment of Broadband over the electricity distribution networks was initiated to exploit the high speed internet capacity, voice over Internet protocol (VOIP) and other broadband application services. This is achieved with the merging of the principles of wireless networking, modems and radios for a wide coverage to the customers and businesses. The entire network system comprises of injector which are interface between the internet backbone and the distribution power lines. The injector are used to inject high frequency signal into the distribution power lines. At the receiver link of the network are the extractors which serve as interface between the end users and the distribution power lines, and help in retrieval of the transmitted signals. Extractors are located by each distribution transformer that serve group of houses around the area. BPL has a coverage 
area between 1000-3000 feet with a good quality of signal at this distance, after this a repeater station can be installed to boost the signal propagation and avoid distortion and total loss of the signal due to propagation and multipath mechanisms [11]

The wide coverage of BPL is as result of the wide coverage of the power lines already in existence virtually everywhere now. This solid structure of the power sector will be a great advantage in taking BPL to rural areas, where communication has been a daunting challenge before, as a result of the presence of already installed power line facility. In the BPL basic architecture, signals are injected into the electric grid from a head end on the Distribution lines at a substation. To back haul the signal to the head end from the substation, fibre or wireless connections are used. The signals traverse the grid network over the distribution lines to the home or business of the end user. Links between the lines are ensured by passing signals either through the transformers or by bypassing the transformer using bridges. Different Vendors such as Amperion inter connect the end users through wireless technology. Repeaters and extractors installation along the line boost the signal and provide customer access [12]. The details of the structure is shown in Fig 1.

\section{DISCUSSION}

The proliferation of the wireless technology and broadband access for real time application of diverse services, and the quest for transmission of digital voice, video and internet data at the comfort of one home and within the neighborhood is on the increase. But it will take a fortune and a lot of time to get this done through special cable fresh fitting of the required facility. This hassle is alleviated by PLC technology that is well suited to be used on the already existed and wide spread low and medium voltage networks infrastructure capable of high speed network transmission and other numerous benefits

It is the longing of internet customer that the digital electronic in the house environment be connected together for easy access of file transfer, information exchange among all these peripheral of the house and appliances. This quest demand wireless internet connectivity in around the house with appreciable distance range of coverage. The structure layout limit the effectiveness of wireless transmission within the building, attenuation is imposed on the transmission in the presence of the household furniture, walls, ceiling and window metals [14]. All these will degrade and reduce that data throughput of a wireless propagation in the house. In the same way, the short wavelength of the operating frequency at high frequency is affected by propagation mechanisms such multipath, scattering, reflection absorption as the signal goes through the free space. It encounters many obstructions that shield the signal from line of sight required by the system. Therefore, the propagation range of such frequency is confined to a room, even at that tangible data rate is not always deliver as a result of obstruction presence, hence the reduced reliability of the network systems. With the emergence of PLC, this challenges can be eroded .This implies that once the laudable project is implemented over $50 \%$ of Nigeria population who currently rely on electricity as a source of power will benefit from cheap and accessible internet services. The Government agenda to extend electricity to $80 \%$ of the population will equally boost the reach of wider audience and especially the rural dwellers with internet connectivity [15]. This on the long run will impart the social economic and political life of the nation and the citizenry as a whole

\section{CONCLUSION}

PLC is gaining wide acceptability today as the technology capable of providing wide range and multimedia application potentials. The technical challenges pose by it is currently under keen attention by the research community. There is no doubt, great advancement and hope is risen on the engagement of the channel to achieve the purpose of digital communication and broad band internet service. There is a huge flow of data traffic in the communication industry today due to avalanche of spectrum of that sustains high definition and multimedia application. This heavy application is preferable on a fixed connection. Since that wireless fixed connection in existence cannot deliver such a huge bandwidth, engaging the established facility of the power line connection will be major feat in the communication industry to achieve this. This means every household already connected to the power grid will have access to ubiquity, clean and regular internet connectivity. The ultimate goal of reaching the rural dwellers with high internet facility will be achieved with affordable cost by the end users. 


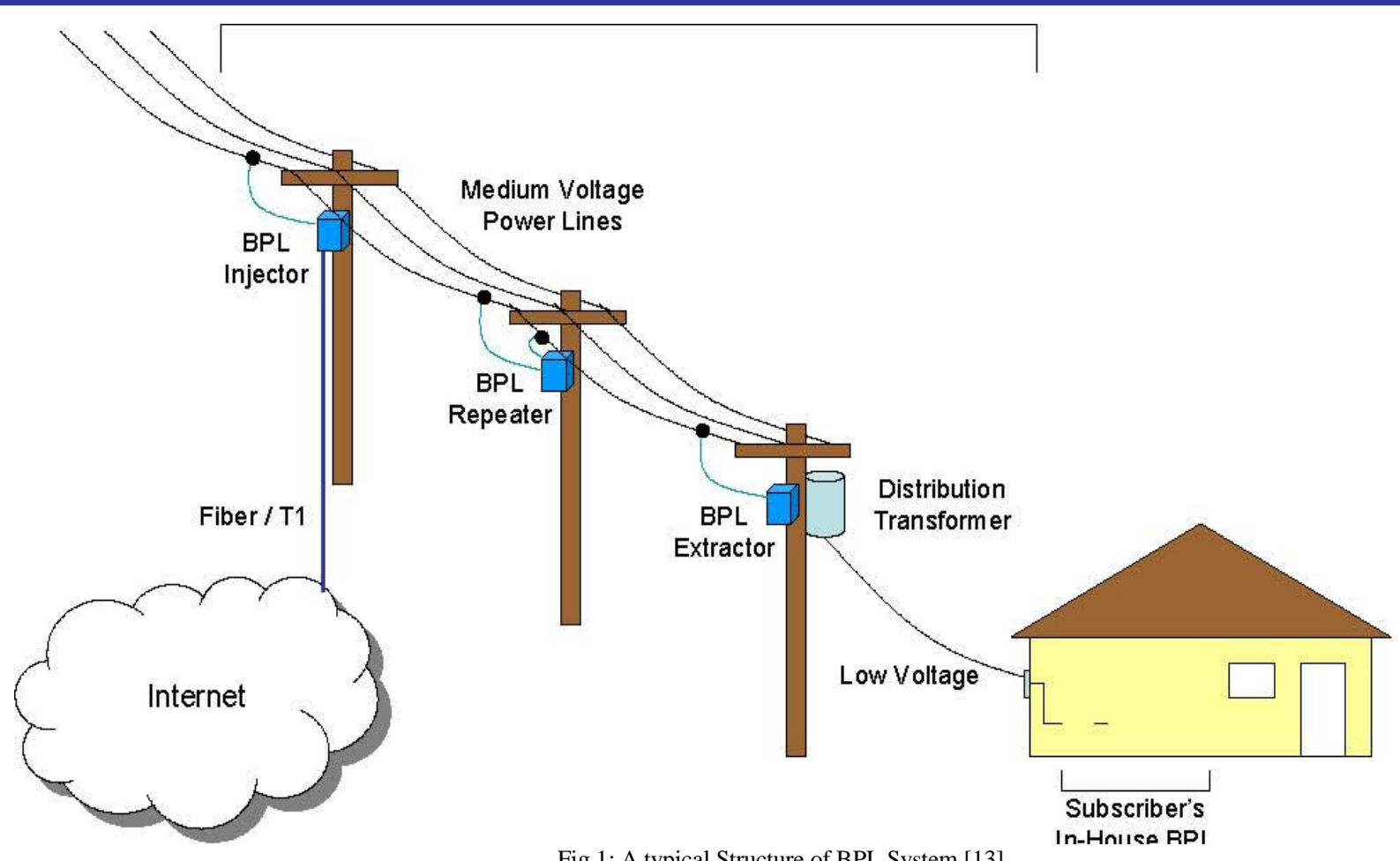

Fig 1: A typical Structure of BPL System [13]

\section{ACKNOWLEDGMENT}

The TetFund Institutional Based Research Scheme, Ekiti State University Chapter is highly appreciated for providing resources for this work and further ones. I like to thank Mr. Uche Emmanuel for his support for this work.

\section{REFERENCES}

[1] Ibikunle Frank*, Jakpa Orunta, Ike Dike Broadband Wireless Access Deployment Approach to Rural Communities Journal of Computer Networks, Vol. 1, No. 3, 38-45 2013, J. Clerk Maxwell, A Treatise on Electricity and Magnetism, 3rd ed., vol. 2. Oxford: Clarendon, 1892, pp.68-73.

[2] Niovi Pavlidou, A. Han Vinck, Javad Yazdani and Bahram Honaty, Power Line Communications: State of the Art and Future Trends IEEE Communications Magazine April 2003I.S. Jacobs and C.P. Bean, "Fine particles, thin films and exchange anisotropy," in Magnetism, vol. III, G.T. Rado and H. Suhl, Eds. New York: Academic, 1963, pp. 271-350.

[3] Mlynek P, Koutyny M, Misurec J Power Line Modelling for Creating Power Line Communication System. Int J Commun 4: 13-21.5 2010K. Elissa, "Title of paper if known," unpublished.

[4] Apena WO, Adebanjo IA Fusing Power Line Communication (PLC) Technologies and Nigerian National Grid Network: Making Sense from Knowledge Management (KM). J Electr Eng Electron Technol 5:3, 2016 R. Nicole, "Title of paper with only first word capitalized," J. Name Stand. Abbrev., in press.

[5] Alberto Sendin , Ivan Peña and Pablo Angueira Strategies for Power Line Communications Smart Metering Network Deployment, Energies, 7, 2377-2420. 2014Y. Yorozu, M. Hirano, K. Oka, and Y. Tagawa, "Electron spectroscopy studies on magneto-optical media and plastic substrate interface,” IEEE Transl. J. Magn. Japan, vol. 2, pp. 740-741,
August 1987 [Digests 9th Annual Conf. Magnetics Japan, p. 301, 1982].

[6] Uduak S. Akpan, Salisu R. Isihak Ye-Obong N. Udoakah Electricity Access in Nigeria: Viability of Off-Grid Photovoltaic System Institute of Electrical and Electronics Engineers (IEEE) African Conference, 2013, M. Young, The Technical Writer's Handbook. Mill Valley, CA University Science, 1989.

[7] Muhammad Salman Yousuf, Mustafa El-Shafei Power Line Communications: An Overview - Part I IEEE 2008

[8] Power Line Communication" [Online]. Available: https://www.cypress.com/applications/power-line-communication [Accessed: 27/03/2019.

[9] Dostert, $\mathrm{K}$ "Telecommunications over the Power Distribution GridPossibilities and Limitations". Proc 1997 Internat. Symp. on Powe Line Comms and its Applications:1-9,1999.

[10] Broadridge, R. Power line modems and networks. Second IEE National Conference on Telecommunications. London UK. pp. 294 296, 1989.

[11] Adekunle, Oluwadara Victoria An Overview of Broadband communication over Power Lines 2nd Intl' Conf.Comp., Energy, Net., Robotics and Telecom Conference pp 118-124, 2014.

[12] Abdul Mannan, D.K.Saxena, Mahroosh Banday A Study on Power Line Communication International Journal of Scientific and Research Publications, Volume 4, Issue 7 pp 2250-2259, 2014.

[13] Omuse Alex Voice And Data Communication Over power Lines Master Thesis at The University Of Nairobi 2009.

[14] Lars T. Berger, Member, IEEE, Andreas Schwager MIMO Power Line Communications http://dx.doi.org/10.1109/COMST 2339276, 2014.

[15] Cristina Cano, Alberto Pittolo, David Malone, Lutz Lampe4 Andrea M Tonello5, Anand Dabak State-of-the-art in Power Line Communications: from the Applications to the Medium arXiv:1602.09019v1 [cs.NI] 29 Feb 2016 